\title{
Editorial
}

\section{Toward a Meta-Analytic Synthesis of the Resting-State fMRI Literature for Clinical Populations}

\author{
Yu-Feng Zang, ${ }^{1,2}$ Xi-Nian Zuo, ${ }^{3}$ Michael Milham, ${ }^{4,5}$ and Mark Hallett ${ }^{6}$ \\ ${ }^{1}$ Center for Cognition and Brain Disorders, Hangzhou Normal University, Hangzhou 310015, China \\ ${ }^{2}$ Zhejiang Key Laboratory for Research in Assessment of Cognitive Impairments, Hangzhou Normal University, \\ Hangzhou 310015, China \\ ${ }^{3}$ Key Laboratory of Behavioral Science and Magnetic Resonance Imaging Research Center, Institute of Psychology, \\ Chinese Academy of Sciences, Beijing 100101, China \\ ${ }^{4}$ Center for the Developing Brain, Child Mind Institute, New York, NY 10022, USA \\ ${ }^{5}$ Center for Biomedical Imaging and Neuromodulation, Nathan S. Kline Institute for Psychiatric Research, \\ Orangeburg, NY 10962, USA \\ ${ }^{6}$ Human Motor Control Section, National Institute of Neurological Disorders and Stroke, NIH, Bethesda, MD 20892, USA \\ Correspondence should be addressed to Yu-Feng Zang; zangyf@gmail.com
}

Received 10 May 2015; Accepted 10 May 2015

Copyright (C) 2015 Yu-Feng Zang et al. This is an open access article distributed under the Creative Commons Attribution License, which permits unrestricted use, distribution, and reproduction in any medium, provided the original work is properly cited.

Coordinate-based meta-analysis (CB-meta) is playing an important role in identifying spatially consistent findings for targeted questions in the neuroimaging literature, by quantitatively aggregating independent results reported in a standard coordinate space [1]. CB-meta has been widely used in voxel-based magnetic resonance imaging (MRI) studies, including task functional MRI (fMRI), where either patterns of within-group activation or patterns of between-group activation differences can be aggregated across studies. Given that variations in task design can introduce confounds into such pursuits, researchers work to limit any effort to the inclusion of studies using highly similar tasks. Task fMRI CBmeta papers have been published in most brain disorders.

Resting-state fMRI (RS-fMRI) does not require cognitive task probes, and its design is inherently similar across studies. From this perspective, it is ideally suited for CBmeta. However, while an array of analytic methods have emerged to characterize different aspects of resting brain activity, not all of them are suitable for CB-meta. This likely explains the relatively small number of RS-fMRI based CBmeta published to date [2-8].

The analytic methods for RS-fMRI can be divided into two categories, one for depicting functional relationships between remote brain regions and another for local activity (for a systematic review, see [9]). The widely used methods in the former category include seed-based functional connectivity, spatial independent component analysis (sICA), and graph theory. The latter has two widely used methods, namely, regional homogeneity ( $\mathrm{ReHo}$ ) and amplitude of low frequency fluctuation (ALFF)/fractional ALFF (fALFF).

Seed-based functional connectivity is one of the most widely used analytic methods in RS-fMRI studies. Typically, a region of interest (ROI) is predefined and then linear correlation or linear regression analysis is performed between the mean time series of this ROI and the time series of each voxel in the brain. The results are of course dependent on the location of seed ROIs and therefore are not suitable for CB-meta. Most of the $8 \mathrm{CB}$-meta RS-fMRI papers aforementioned did not include seed-based functional connectivity studies.

Spatial independent component analysis (sICA) decomposes the RS-fMRI data into multiple networks (components), among which only about 10 networks are psychophysiologically interpretable [10]. "Spatial independent" means spatially nonoverlapping. Therefore, sICA papers should not be taken into a CB-meta study, unless different papers have focused on the same component (e.g., the default mode network). A few CB-meta RS-fMRI studies have included 
sICA RS-fMRI papers [2, 4, 6, 8]. However, few explicitly mentioned the limitation of sICA for CB-meta.

Graph theory has been widely used for exploring the topological organization of complex brain networks in RSfMRI studies. Unlike seed-based functional connectivity and sICA, which focus on a specific functional system(s), graph theory usually explores the topological properties, such as small-world, modular structure and highly connected hubs, of the entire brain. From this point of view, RS-fMRI studies using graph theory are also well suitable for meta-analysis. However, due to the high computational cost, most graphbased RS-fMRI studies have chosen a limited number of brain regions or ROIs, rather than brain voxels, as network nodes. Such results do not provide the coordinate information needed to support CB-meta. Degree centrality is one of the simplest and least computation-demanding measures for graph theory complex networks. Several RS-fMRI toolboxes (e.g., DPARSF (http://www.restfmri.net/; http://rfmri.org/) and Gretna (https://www.nitrc.org/projects/gretna/)) have included voxel-based degree centrality measurement [11, 12]. Some studies have applied voxel-based centrality to brain disorders including depression [13-15] and Alzheimer's disease [16]. In light of recent increases in computational capacity, more complicated measurements of graph theory will be implemented in a voxel-wise manner, thereby increasing the suitability of graph theory for CB-meta.

ReHo and ALFF are two methods widely used for characterizing local spontaneous activity of RS-fMRI data. ReHo measures the local synchronization of the time series of neighboring voxels [17] whereas ALFF/fALFF measures the amplitude of time series fluctuations at each voxel [18, 19]. Although both ReHo and ALFF/fALFF measure the local activity of each voxel, many studies used the two measurements and suggested that the two methods reveal different aspects of brain function and abnormalities arising in clinical populations [20-23]. For nearly all of the existing CB-meta RS-fMRI studies, researchers have combined across these methods for characterizing local activity (ReHo and/or ALFF), despite known differences in their properties. A CBmeta RS-fMRI study of depression by Iwabuchi and colleagues for the first time included RS-fMRI papers using the same analytic method (ReHo) [5]. This approach markedly reduces discrepancies in analytic methods. However, only 10 of the $200+$ RS-fMRI papers on depression to date met the inclusion criteria of that CB-meta study.

Clinical studies always face the challenges of high heterogeneity and limited sample size in patient groups. Therefore, meta-analysis is critical for drawing congruent conclusion across studies carried out with similar settings and techniques. Few techniques for clinical studies enable the application of the broad range of analytic methods that RSfMRI does in an effort to reveal the functional complexity of the human brain from multiple aspects. Although some analytic methods are not suitable for CB-meta, the strength of RS-fMRI is that its design is inherently similar across studies. Therefore, each dataset could be reanalyzed by using analytic methods being suitable to perform CB-meta. The current special issue was launched to encourage RS-fMRI studies on brain disorders by reanalyzing the published data with methods supporting future CB-meta.

In the current issue, a paper from Dr. Chunshui Yu's group in Tianjin Medical University is of particular interest (see Y. Xu et al., "Altered Spontaneous Brain Activity in Schizophrenia: A Meta-Analysis and a Large-Sample Study"). The authors not only performed two CB-meta RS-fMRI studies, in which only RS-fMRI papers using methods for local activity (ReHo and ALFF, resp.) were included, but they also validated the CB-meta results in their own dataset obtained from a relatively large sample of schizophrenia patients. One of the congruent results was that ALFF was reduced in the primary visual and primary sensorimotor cortex (see details in Y. Xu et al. "Altered Spontaneous Brain Activity in Schizophrenia: A Meta-Analysis and a LargeSample Study"). The limitation of this study, like other CBmeta RS-fMRI studies, is the small number of eligible RSfMRI papers. Only 6 ALFF papers and 4 ReHo RS-fMRI papers were included.

We hope this special issue will draw attention to the need for and value of CB-meta in the RS-fMRI research field. If your dataset has not been analyzed with a method that facilitates future CB-meta, please try it. Future CB-meta efforts need large numbers of studies for inclusion to enable more definitive conclusions, upon which models for the prediction or diagnosis of brain disorders can be developed. Additionally, accurate localization of abnormal spontaneous brain activity may help to guide intervention therapies (e.g., deep brain stimulation, transcranial magnetic stimulation, or transcranial ultrasound stimulation).

\section{Acknowledgments}

Dr. Yu-Feng Zang was supported by NSFC (31471084 and 81271652) and "Qian Jiang Distinguished Professor" program. Dr. Xi-Nian Zuo acknowledges the Hundred Talents Program and the Key Research Program of the Chinese Academy of Sciences (KSZD-EW-TZ-002). Dr. Michael Milham is supported by awards from the NIMH (R01 MH094639, U01MH099059), as well as gifts from Phyllis and Randolph Cowen, and Joseph Healey to the Child Mind Institute. Dr. Mark Hallett is supported by the NINDS Intramural Program. We thank Dr. Yong HE (yong.h.he@gmail.com) for editing the graph theory paragraph.

Yu-Feng Zang
Xi-Nian Zuo
Michael Milham
Mark Hallett

\section{References}

[1] S. B. Eickhoff, A. R. Laird, C. Grefkes, L. E. Wang, K. Zilles, and P. T. Fox, "Coordinate-based activation likelihood estimation meta-analysis of neuroimaging data: a random-effects approach based on empirical estimates of spatial uncertainty," Human Brain Mapping, vol. 30, no. 9, pp. 2907-2926, 2009.

[2] S. Kühn and J. Gallinat, "Resting-state brain activity in schizophrenia and major depression: a quantitative metaanalysis," Schizophrenia Bulletin, vol. 39, no. 2, pp. 358-365, 2013. 
[3] J. Graham, G. Salimi-Khorshidi, C. Hagan et al., "Meta-analytic evidence for neuroimaging models of depression: state or trait?" Journal of Affective Disorders, vol. 151, no. 2, pp. 423-431, 2013.

[4] B. Sundermann, M. L. Beverborg, and B. Pfleiderer, "Toward literature-based feature selection for diagnostic classification: a meta-analysis of resting-state fMRI in depression," Frontiers in Human Neuroscience, vol. 8, article 692, 2014.

[5] S. J. Iwabuchi, R. Krishnadas, C. Li, D. P. Auer, J. Radua, and L. Palaniyappan, "Localized connectivity in depression: a meta-analysis of resting state functional imaging studies," Neuroscience \& Biobehavioral Reviews, vol. 51, pp. 77-86, 2015.

[6] S. M. Palmer, S. G. Crewther, L. M. Carey, and START Project Team, "A meta-analysis of changes in brain activity in clinical depression," Journal of Frontiers in Human Neuroscience, vol. 8, article 1045, 2015.

[7] Z. Q. Chen, M. Y. Du, Y. J. Zhao et al., "Voxel-wise metaanalyses of brain blood flow and local synchrony abnormalities in medication-free patients with major depressive disorder," Journal of Psychiatry \& Neuroscience, vol. 40, no. 2, Article ID 140119, 2015.

[8] Y. Hannawi, M. A. Lindquist, B. S. Caffo, H. I. Sair, and R. D. Stevens, "Resting brain activity in disorders of consciousness: a systematic review and meta-analysis," Neurology, vol. 84, no. 12, pp. 1272-1280, 2015.

[9] X. Zuo and X. Xing, "Test-retest reliabilities of resting-state FMRI measurements in human brain functional connectomics: a systems neuroscience perspective," Neuroscience \& Biobehavioral Reviews, vol. 45, pp. 100-118, 2014.

[10] J. S. Damoiseaux, S. A. R. B. Rombouts, F. Barkhof et al., "Consistent resting-state networks across healthy subjects," Proceedings of the National Academy of Sciences of the United States of America, vol. 103, no. 37, pp. 13848-13853, 2006.

[11] Y. Chao-Gan and Z. Yu-Feng, "DPARSF: a MATLAB toolbox for 'pipeline' data analysis of resting-state fMRI," Frontiers in System Neuroscience, vol. 4, article 13, 2010.

[12] T. Xu, Z. Yang, L. Jiang, X.-X. Xing, and X.-N. Zuo, "A connectome computation system for discovery science of brain," Science Bulletin, vol. 60, no. 1, pp. 86-95, 2015.

[13] L. Wang, Z. Dai, H. Peng et al., "Overlapping and segregated resting-state functional connectivity in patients with major depressive disorder with and without childhood neglect," Human Brain Mapping, vol. 35, no. 4, pp. 1154-1166, 2014.

[14] L. Wang, M. Xia, K. Li et al., "The effects of antidepressant treatment on resting-state functional brain networks in patients with major depressive disorder," Human Brain Mapping, vol. 36, no. 2, pp. 768-778, 2015.

[15] Y. Shen, J. Yao, X. Jiang et al., "Sub-hubs of baseline functional brain networks are related to early improvement following twoweek pharmacological therapy for major depressive disorder," Human Brain Mapping, 2015.

[16] Z. Dai, C. Yan, K. Li et al., "Identifying and mapping connectivity patterns of brain network hubs in Alzheimer's disease," Cerebral Cortex, 2014.

[17] Y. Zang, T. Jiang, Y. Lu, Y. He, and L. Tian, "Regional homogeneity approach to fMRI data analysis," NeuroImage, vol. 22, no. 1 , pp. 394-400, 2004.

[18] Y. F. Zang, Y. He, C. Z. Zhu et al., "Altered baseline brain activity in children with ADHD revealed by resting-state functional MRI," Brain \& Development, vol. 29, no. 2, pp. 83-91, 2007.

[19] Q. H. Zou, C. Z. Zhu, Y. Yang et al., "An improved approach to detection of amplitude of low-frequency fluctuation (ALFF) for resting-state fMRI: fractional ALFF,' Journal of Neuroscience Methods, vol. 172, no. 1, pp. 137-141, 2008.

[20] D. Lei, J. Ma, X. Du, G. Shen, M. Tian, and G. Li, "Spontaneous brain activity changes in children with primary monosymptomatic nocturnal enuresis: a resting-state fMRI study," $\mathrm{Neu}$ rourology and Urodynamics, vol. 31, no. 1, pp. 99-104, 2012.

[21] L. An, Q.-J. Cao, M.-Q. Sui et al., "Local synchronization and amplitude of the fluctuation of spontaneous brain activity in attention-deficit/hyperactivity disorder: a resting-state fMRI study," Neuroscience Bulletin, vol. 29, no. 5, pp. 603-613, 2013.

[22] E. Premi, F. Cauda, R. Gasparotti et al., "Multimodal FMRI resting-state functional connectivity in Granulin mutations: the case of fronto-parietal dementia," PLoS ONE, vol. 9, no. 9, Article ID e106500, 2014.

[23] L. Han, L. Zhaohui, Y. Fei et al., "Disrupted neural activity in unilateral vascular pulsatile tinnitus patients in the early stage of disease: evidence from resting-state fMRI," Progress in NeuroPsychopharmacology and Biological Psychiatry, vol. 59, pp. 9199, 2015. 

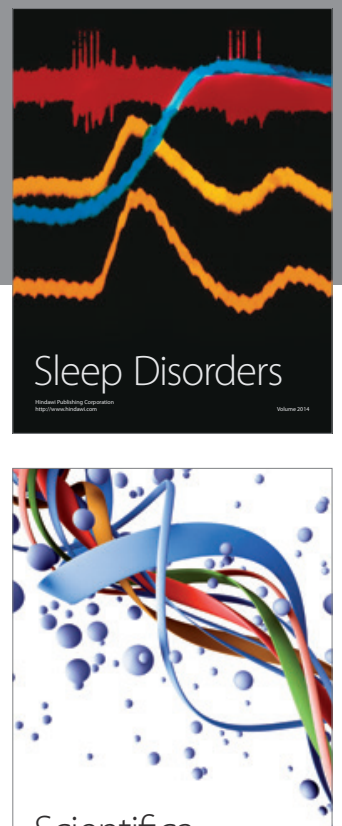

Scientifica
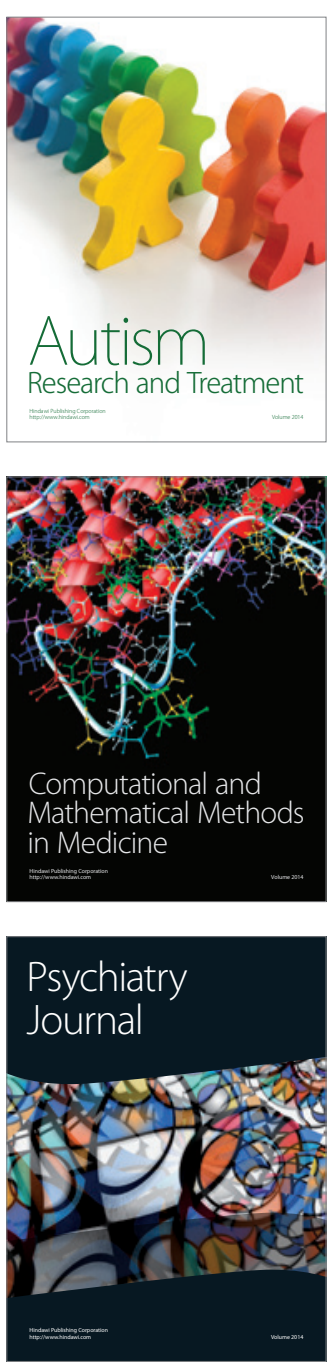
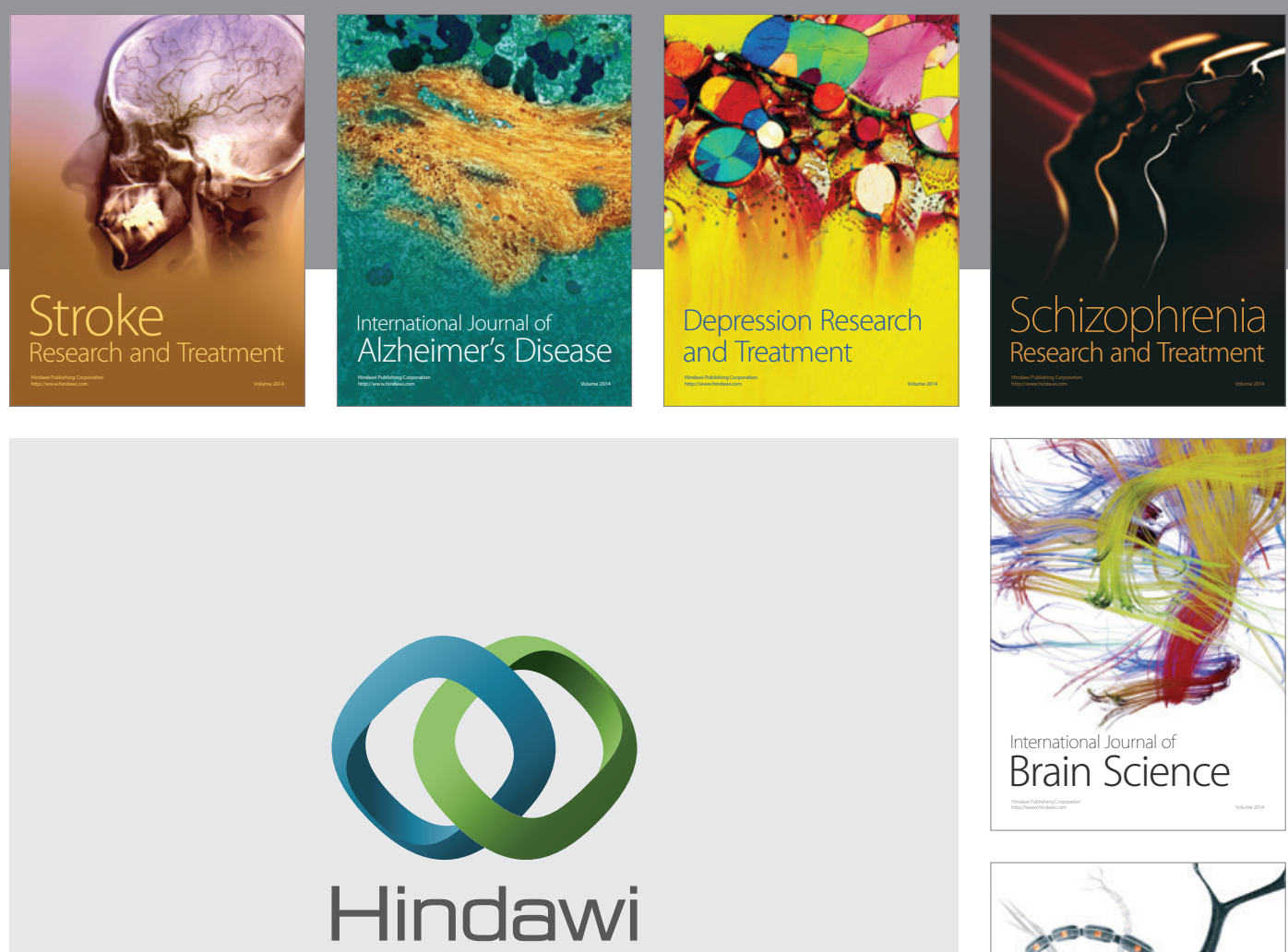

Submit your manuscripts at

http://www.hindawi.com
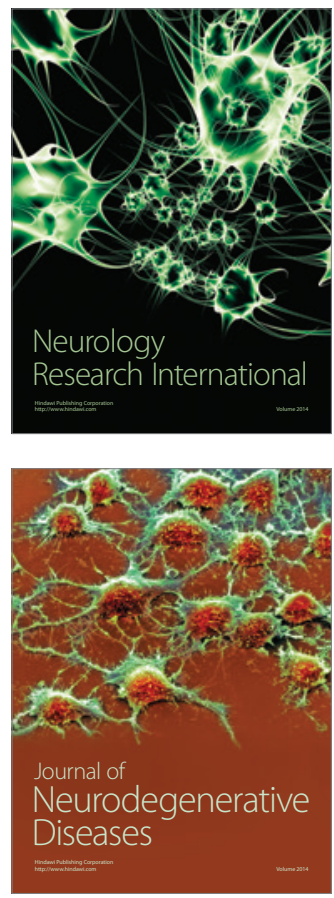

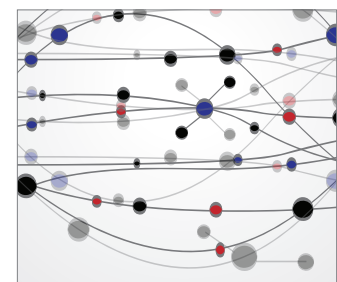

The Scientific World Journal
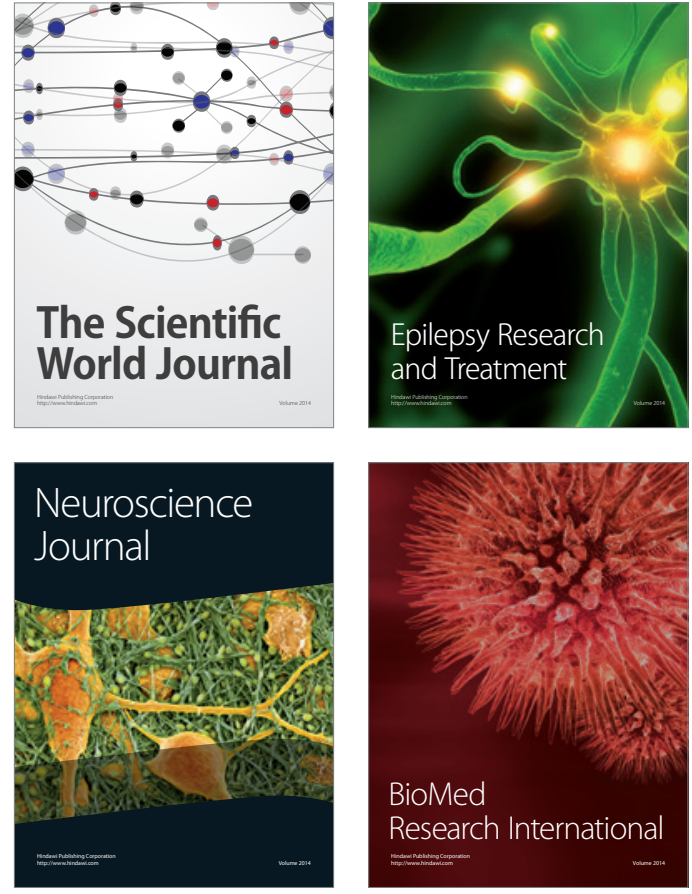

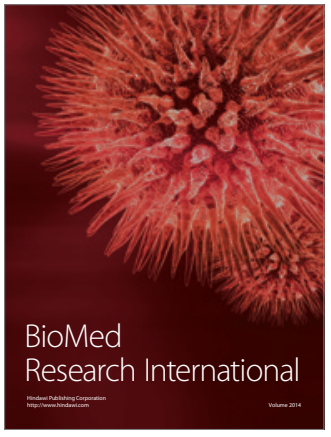

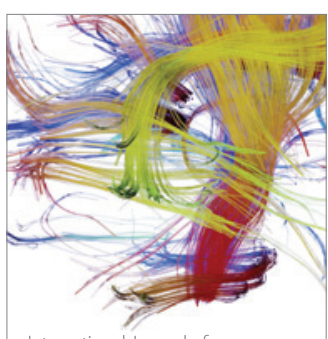

Brain Science

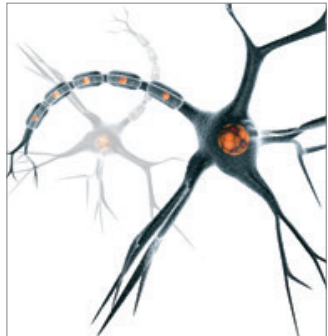

Neural Plasticity
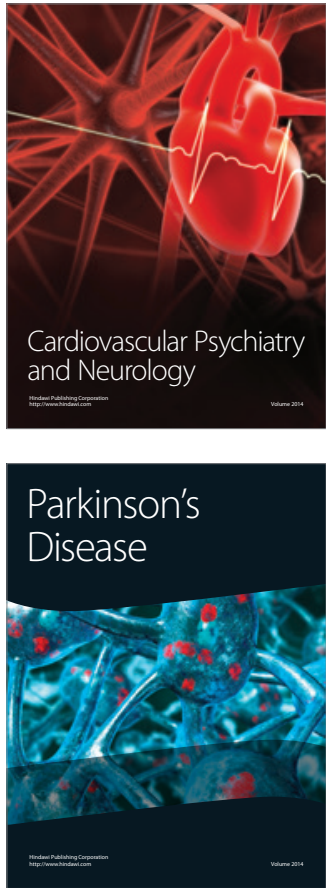